\title{
A 100 pc ELLIPTICAL AND TWISTED RING OF COLD AND DENSE MOLECULAR CLOUDS REVEALED BY HERSCHEL AROUND THE GALACTIC CENTER
}

\author{
S. Molinari ${ }^{1}$, J. Bally ${ }^{2}$, A. Noriega-Crespo ${ }^{3}$, M. Compiègne $^{3}$, J. P. Bernard ${ }^{4,5}$, D. Paradis ${ }^{4}$, P. Martin ${ }^{6}$, L. Testi ${ }^{7,8}$, \\ M. Barlow ${ }^{9}$, T. Moore ${ }^{10}$, R. Plume ${ }^{11}$, B. Swinyard ${ }^{9,12}$, A. Zavagno ${ }^{13}$, L. Calzoletti ${ }^{14}$, A. M. Di Giorgio ${ }^{1}$, D. Elia ${ }^{1}$, \\ F. Faustini ${ }^{14}$, P. Natoli ${ }^{14,15}$, M. Pestalozzi ${ }^{1}$, S. Pezzuto ${ }^{1}$, F. Piacentini ${ }^{16}$, G. Polenta ${ }^{14}$, D. Polychroni ${ }^{1}$, E. Schisano ${ }^{1}$, \\ A. Traficante ${ }^{17}$, M. Veneziani ${ }^{16}$, C. Battersby ${ }^{2}$, M. Burton ${ }^{18}$, S. CAREY ${ }^{3}$, Y. Fukui ${ }^{19}$, J. Z. Li $^{20}$, \\ S. D. Lord $^{21}$, L. Morgan ${ }^{10}$, F. Motte ${ }^{22}$, F. Schuller ${ }^{23}$, G. S. Stringfellow ${ }^{2}$, J. C. TAN ${ }^{24}$, M. A. Thompson ${ }^{25}$, \\ D. WARD-ThOMPSON ${ }^{26}$, G. White ${ }^{12,27}$, AND G. UMANA ${ }^{28}$ \\ ${ }^{1}$ INAF-Istituto Fisica Spazio Interplanetario, Via Fosso del Cavaliere 100, I-00133 Roma, Italy \\ ${ }^{2}$ Center for Astrophysics and Space Astronomy, University of Colorado, Boulder, CO 80309, USA \\ ${ }^{3}$ Spitzer Science Center, California Institute of Technology, 1200 E. California B1., Pasadena, CA 91125, USA \\ ${ }^{4}$ CNRS, IRAP, 9 Av. colonel Roche, BP 44346, F-31028 Toulouse cedex 4, France \\ ${ }^{5}$ Université de Toulouse, UPS-OMP, IRAP, F-31028 Toulouse cedex 4, France \\ ${ }^{6}$ Department of Astronomy \& Astrophysics, University of Toronto, Canada \\ ${ }^{7}$ European Southern Observatory, Karl Schwarzschild Str. 2, 85748 Garching, Germany \\ ${ }^{8}$ INAF-Osservatorio Astrofisico di Arcetri, Largo E. Fermi 5, 50125 Firenze, Italy \\ ${ }^{9}$ Department of Physics and Astronomy, University College London, London, UK \\ ${ }^{10}$ Astrophysics Research Institute, Liverpool John Moores University, UK \\ ${ }^{11}$ Department of Physics \& Astronomy, University of Calgary, Canada \\ ${ }^{12}$ STFC, Rutherford Appleton Labs, Didcot, UK \\ ${ }^{13}$ LAM, Université de Provence, Marseille, France \\ ${ }^{14}$ Agenzia Spaziale Italiana (ASI) Science Data Center, I-00044 Frascati (Roma), Italy \\ ${ }^{15}$ Dipartimento di Fisica, Università di Ferrara, Ferrara, Italy \\ ${ }^{16}$ Dipartimento di Fisica, Università di Roma 1 "La Sapienza," Roma, Italy \\ ${ }^{17}$ Dipartimento di Fisica, Università di Roma 2 "Tor Vergata," Roma, Italy \\ ${ }^{18}$ School of Physics, University of New South Wales, Australia \\ ${ }^{19}$ Department of Astrophysics, Nagoya University, Nagoya, Japan \\ ${ }^{20}$ National Astronomical Observatories, Chinese Academy of Sciences, Beijing, China \\ ${ }^{21}$ NASA Herschel Science Center-California Institute of Technology, 1200 E. California B1., Pasadena, CA 91125, USA \\ ${ }^{22}$ Laboratoire AIM, CEA/DSM-INSU/CNRS-Université Paris Diderot, IRFU/SAp CEA-Saclay, 91191 Gif-sur-Yvette, France \\ ${ }^{23}$ MPIfR-MPG, Bonn, Germany \\ ${ }^{24}$ Departments of Astronomy \& Physics, University of Florida, Gainesville, FL 32611, USA \\ ${ }^{25}$ Centre for Astrophysics Research, Science and Technology Research Institute, University of Hertfordshire, Hatfield, UK \\ ${ }^{26}$ School of Physics and Astronomy, Cardiff University, Cardiff, UK \\ 27 Department of Physics and Astronomy, The Open University, Milton Keynes, UK \\ ${ }^{28}$ INAF-Osservatorio Astrofisico di Catania, Catania, Italy \\ Received 2011 February 14; accepted 2011 May 17; published 2011 June 21
}

\begin{abstract}
Thermal images of cold dust in the Central Molecular Zone of the Milky Way, obtained with the far-infrared cameras on board the Herschel satellite, reveal a $\sim 3 \times 10^{7} M_{\odot}$ ring of dense and cold clouds orbiting the Galactic center. Using a simple toy model, an elliptical shape having semi-major axes of 100 and $60 \mathrm{pc}$ is deduced. The major axis of this $100 \mathrm{pc}$ ring is inclined by about $40^{\circ}$ with respect to the plane of the sky and is oriented perpendicular to the major axes of the Galactic Bar. The $100 \mathrm{pc}$ ring appears to trace the system of stable $x_{2}$ orbits predicted for the barred Galactic potential. Sgr $\mathrm{A}^{\star}$ is displaced with respect to the geometrical center of symmetry of the ring. The ring is twisted and its morphology suggests a flattening ratio of 2 for the Galactic potential, which is in good agreement with the bulge flattening ratio derived from the 2MASS data.
\end{abstract}

Key words: Galaxy: center - ISM: clouds - stars: formation

\section{INTRODUCTION}

Observations of the central regions of our Milky Way provide the nearest template for the study of the conditions in galactic nuclei in normal galaxies. The $3.6 \times 10^{6} M_{\odot}$ black hole at the center dominates the Central Molecular Zone (CMZ) which extends from Galactic longitude $l=-1^{\circ}$ to about +1.5 and hosts the densest and most massive molecular clouds in the Milky Way (Morris \& Serabyn 1996). The CMZ may be a Galactic analog of the nuclear star-forming rings observed in the centers of star-forming barred galaxies (Kormendy \& Kennicutt 2004; Kormendy \& Cornell 2004). The CMZ contains tens of millions of solar masses of cold interstellar matter (ISM; Pierce-Price et al. 2000; Bally et al. 2010) and harbors several of its most active sites of star formation such as Sgr A, Sgr B2, and Sgr C (Yusef-Zadeh et al. 2008, 2009). The gas kinematics and distribution of near-infrared light indicate that the gravitational potential in the inner Galaxy is dominated by a bar with a major axis inclined by about $20^{\circ}-45^{\circ}$ with respect to our line of sight (Binney et al. 1991; Benjamin et al. 2003; Minchev et al. 2007).

Despite the active star formation and bar dynamics, warm dust has been found to be a relative minor constituent in the CMZ. Sodroski et al. (1994) used COBE/DIRBE data to constrain dust properties in the CMZ and Rodríguez-Fernández et al. (2004) presented Infrared Space Observatory observations. 


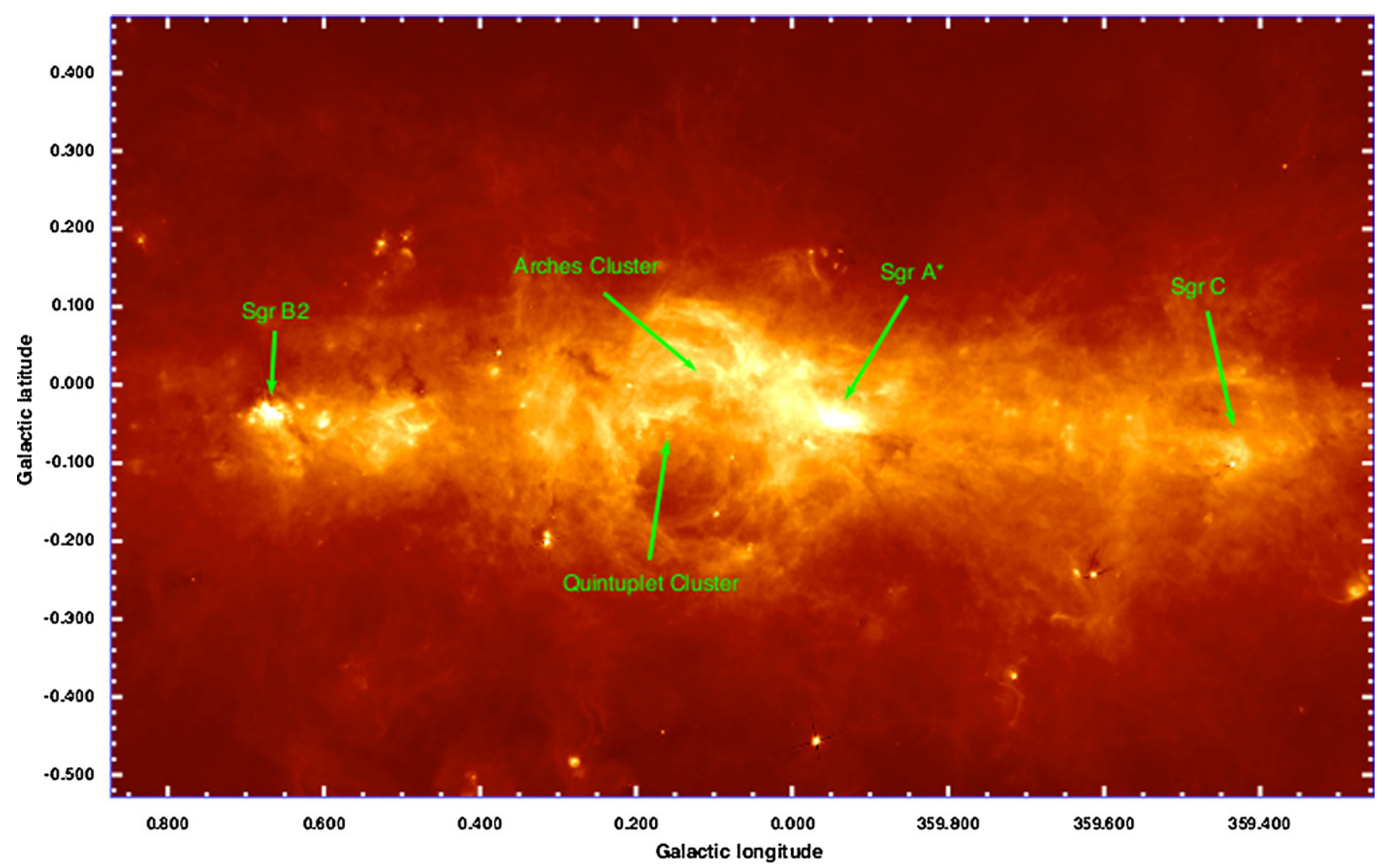

Figure 1. Herschel PACS $70 \mu \mathrm{m}$ image of the Galactic center region. Labels identify known objects that are discussed in the text.

These studies found that $15 \%-30 \%$ of the far-IR emission comes from molecular clouds with dust temperatures of about $19 \mathrm{~K}$, $70 \%-75 \%$ arises from the $\mathrm{H}$ i phase with dust temperatures of $17-22 \mathrm{~K}$, and less than $10 \%$ is emitted by warm dust with $T>$ $29 \mathrm{~K}$ associated with the extended $\mathrm{H}$ II and warm (100-300 K) molecular phases traced by $\mathrm{H}_{3}^{+}$absorption (Oka et al. 2005; Goto et al. 2008). With Herschel ${ }^{29}$ we can now extend this type of analysis into a new domain of spatial resolution and sensitivity.

In this Letter, we present the first high angular resolution maps of the CMZ far-IR dust emission, temperature, and column density based on data obtained with the Herschel satellite (Pilbratt et al. 2010).

\section{OBSERVATIONS AND RESULTS}

The Galactic center was observed with Herschel as part of the Hi-GAL Key-Project (the Herschel infrared Galactic Plane Survey; Molinari et al. 2010a). Observing strategy and data reduction are described in Molinari et al. (2010b) and Traficante et al. (2011). The central $2^{\circ} \times 2^{\circ}$ region of the Galaxy was imaged in Parallel Mode by simultaneously acquiring Photodetector Array Camera and Spectrometer (PACS; Poglitsch et al. 2010) and Spectral and Photometric Imaging REceiver (SPIRE; Griffin et al. 2010) images in five photometric bands centered at 70, 160, 250, 350, and $500 \mu \mathrm{m}$. SPIRE was used in "bright-source mode" to minimize nonlinearities and saturation, and preliminary SPIRE calibration checks indicate an

\footnotetext{
29 Herschel is an ESA space observatory with science instruments provided by European-led Principal Investigator consortia and with important participation from NASA.
}

accuracy within $15 \%$ compared to standard SPIRE setting (M. Pohlen 2010, private communication). We corrected the zero level in our SPIRE and PACS images using offset values derived from the comparison with the Planck/IRAS data using the same procedure as in Bernard et al. (2010).

Figure 1 shows the PACS $70 \mu \mathrm{m}$ image. Sgr $\mathrm{A}^{\star}$ and Sgr B2 are the brightest spots, and the entire area is filled with intense far-IR emission punctuated by several InfraRed Dark Clouds (IRDCs) seen in silhouette. A large bubble dominates at $70 \mu \mathrm{m}$ from $0^{\circ} \lesssim l \lesssim+0^{\circ} 20$ but disappears at longer wavelengths (see SPIRE $250 \mu \mathrm{m}$ in Figure 2). At $250 \mu \mathrm{m}$, Sgr B2 is the brightest feature (it is saturated at $250 \mu \mathrm{m}$ ). Two bent chains of emission extend above and below the mid-plane from Sgr B2 to $\operatorname{Sgr} A^{\star}$, where they appear to cross, and continue on to Sgr C. This structure can also be identified in the SCUBA map (Pierce-Price et al. 2000), but only the unique multi-band far-IR/submillimeter capabilities of the Herschel PACS and SPIRE cameras make possible the determination of its temperature and column density.

Opacity and dust temperature are estimated from a pixelto-pixel fit to the $70-350 \mu \mathrm{m}$ data (the resolution is matched to the $350 \mu \mathrm{m}$ images; $\sim 25^{\prime \prime}$ ) using the DustEM model (Compiègne et al. 2011) following the method described in Bernard et al. (2010). Assuming constant dust properties, the opacity is converted into a hydrogen column density $\left(N_{\mathrm{H}}=\right.$ $N_{\mathrm{HI}+N_{\mathrm{H}_{2}}}$ ) using $\tau_{250} / N_{\mathrm{H}}=8.8 \times 10^{-26} \mathrm{~cm}^{2} / \mathrm{H}$. This value is estimated from latitudes $b>0$, and it could be higher by a factor 2-4 in the Galactic plane (Bernard et al. 2010) and could change our mass estimates accordingly. The temperature and column density maps (Figures 3 and 4) represent the integrated contribution of dust along the line of sight. High opacity, 


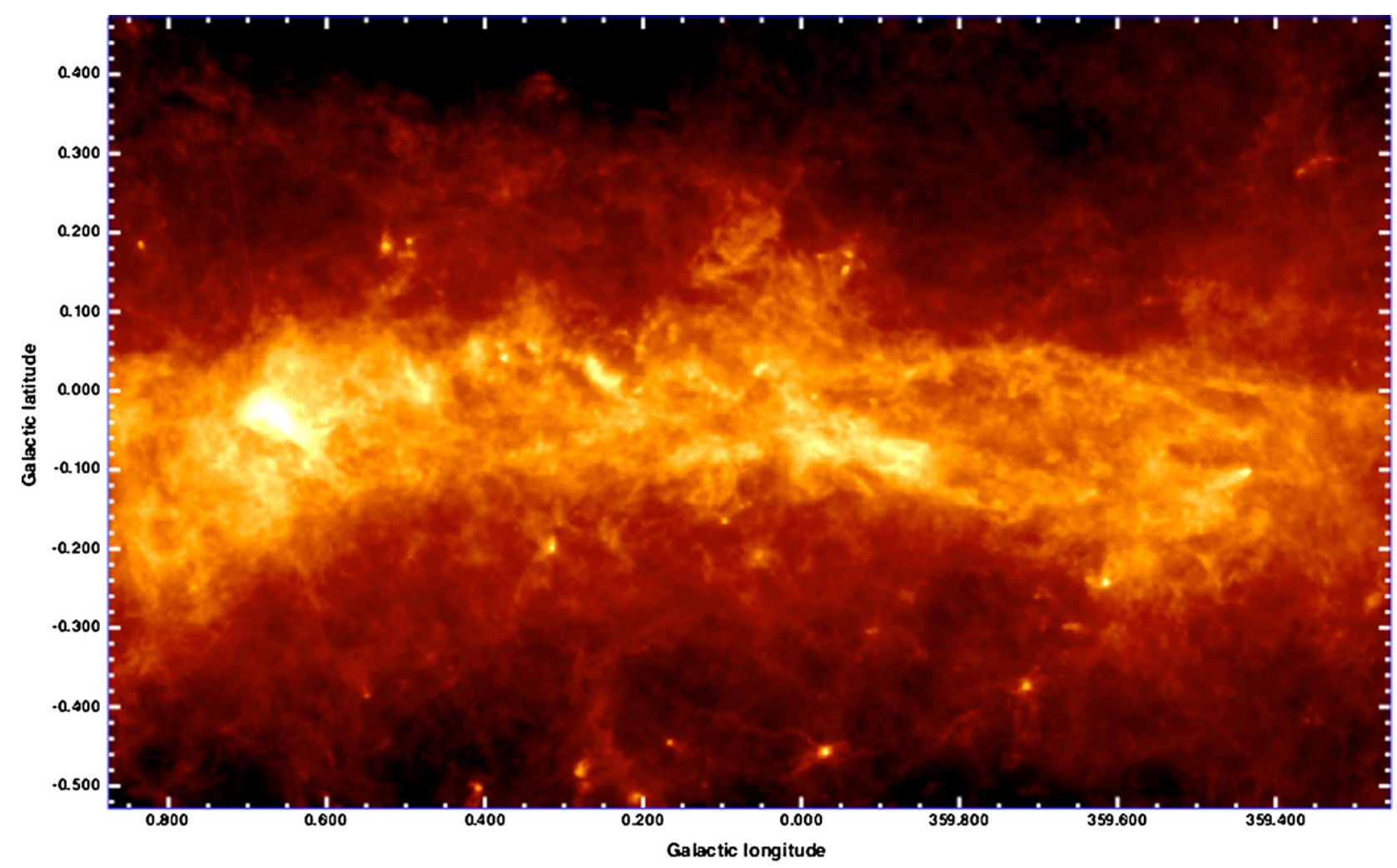

Figure 2. Herschel SPIRE $250 \mu \mathrm{m}$ image of the Galactic center region.

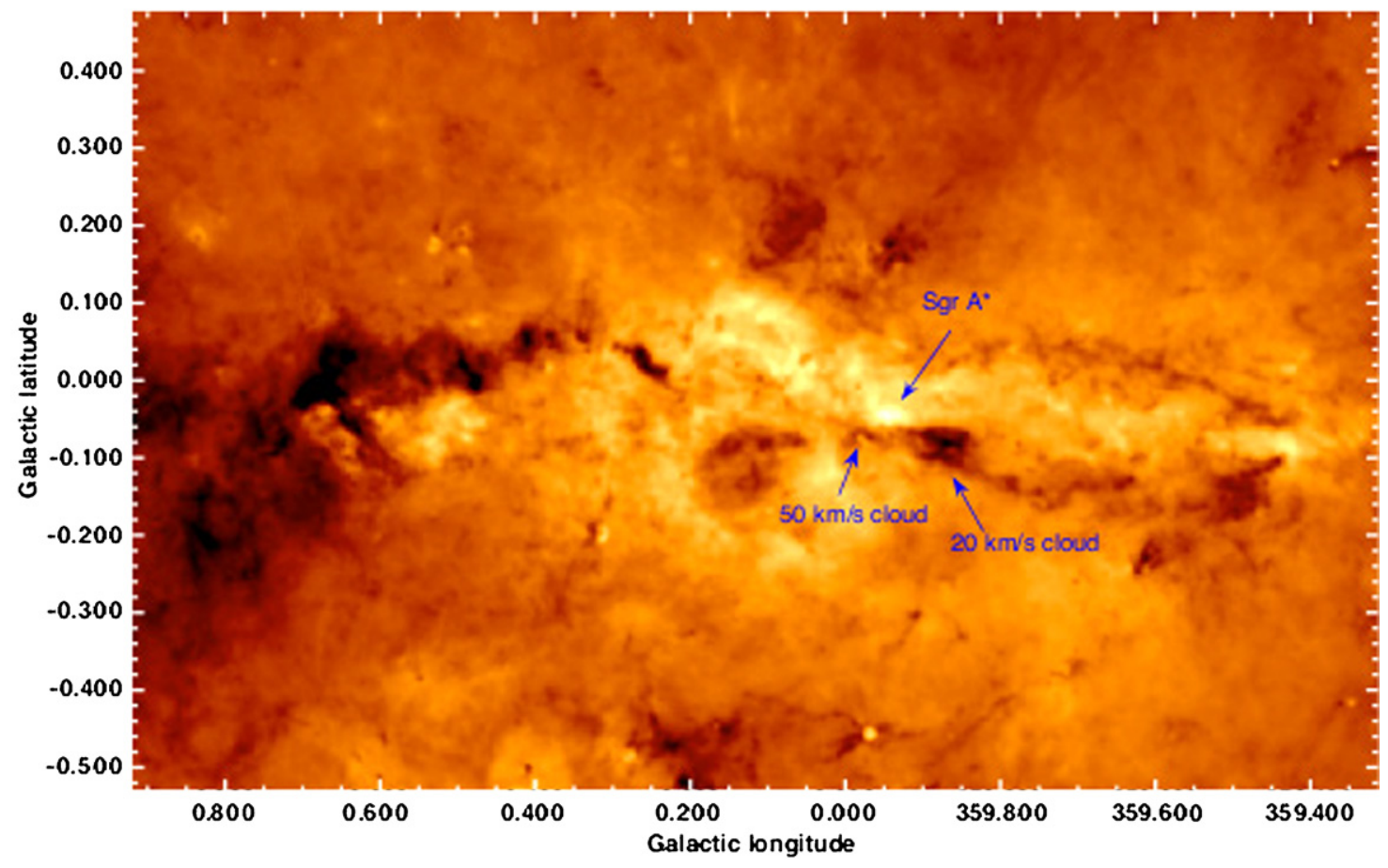

Figure 3. Temperature map of the Galactic center region. The log-color stretch extends from $15 \mathrm{~K}$ to $40 \mathrm{~K}$ on $\mathrm{Sgr} \mathrm{A}^{\star}$.

cold dust appears in silhouette against the warmer background even at $70 \mu \mathrm{m}$ (Figure 1). Although the derived temperature might be underestimated, a temperature map without the $70 \mu \mathrm{m}$ data provides similar results.

The detailed study of the great bubble visible in the $70 \mu \mathrm{m}$ and the temperature maps (Figures 1 and 3) will be presented in a forthcoming paper. This Letter is dedicated to the analysis of the large-scale filaments visible in the far-IR (Figure 2) that trace a continuous chain of cold and dense clumps $\left(T_{\text {dust }} \leqslant 20 \mathrm{~K}\right.$, $N(\mathrm{H})>2 \times 10^{23} \mathrm{~cm}^{-2}$ ) organized along an $\infty$-shaped feature that dominates the image between the Sgr C complex at $l=$ $359^{\circ} 4$ and Sgr B2 at 0.7. The total projected extent is $\sim 1.4$, or about $180 \mathrm{pc}$, for a solar Galactocentric distance of $8.4 \mathrm{kpc}$ (Reid et al. 2009). Warmer dust with relatively lower column density $\left(T_{\text {dust }} \geqslant 25 \mathrm{~K}, N(\mathrm{H})<2 \times 10^{23} \mathrm{~cm}^{-2}\right)$ fills the interior of the $\infty$-shaped feature. 


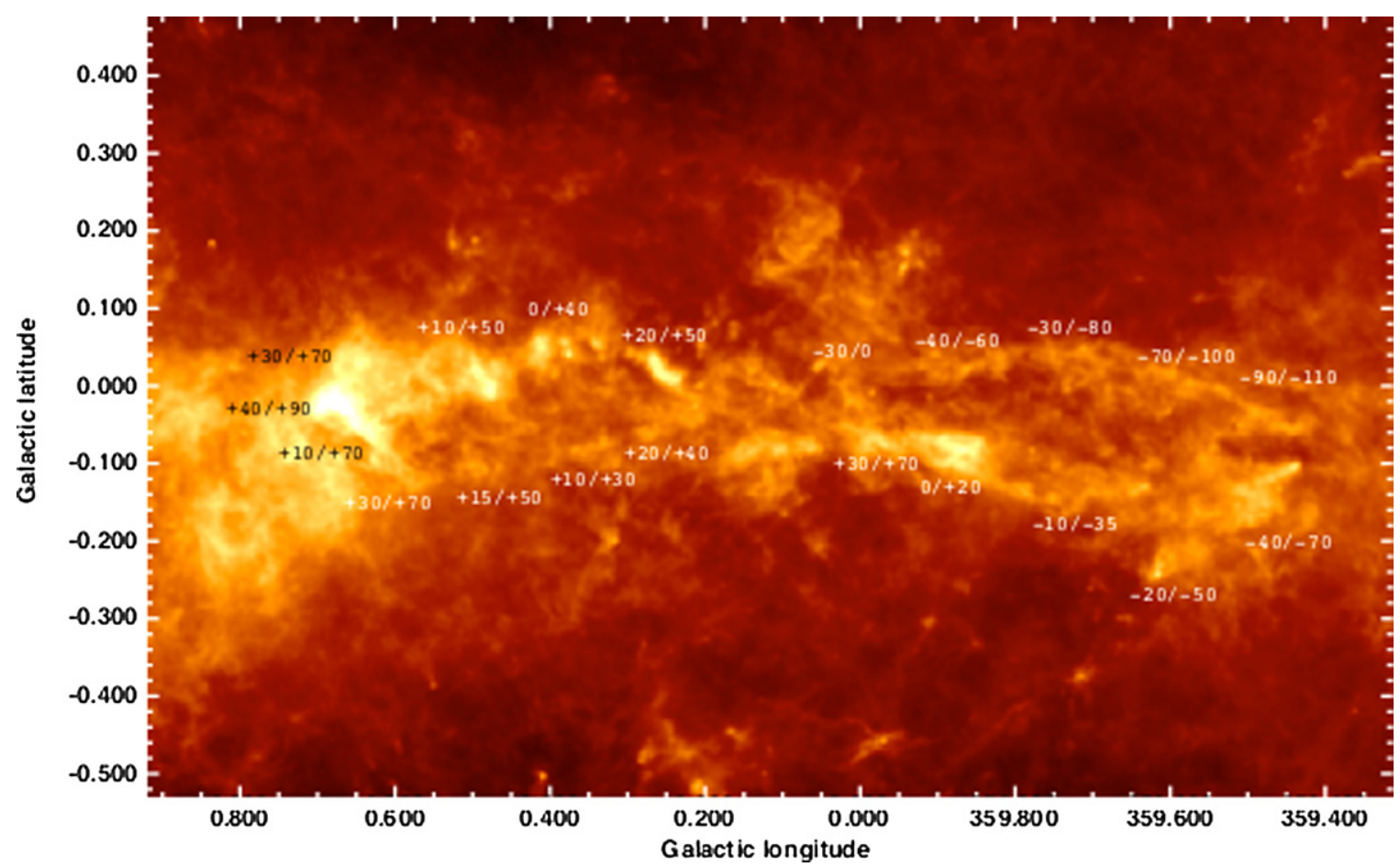

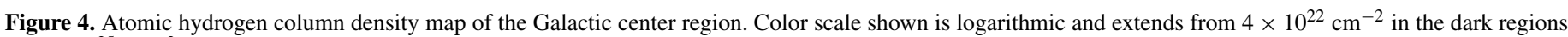

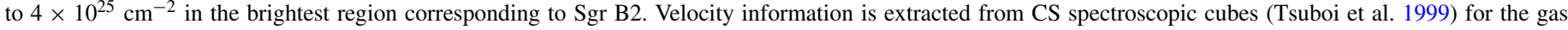
counterparts positionally associated with the dense dust clumps; the range values indicate the range where emission is observed.

\section{A $100 \mathrm{pc}$ ELLIPTICAL RING OF COLD AND DENSE MOLECULAR CLOUDS: THE $x_{2}$ ORBITS}

The $\infty$-shaped feature in the temperature map in Figure 3 coincides with high column density material $(N(\mathrm{H}) \geqslant 2 \times$ $10^{23} \mathrm{~cm}^{-2}$ ) organized in a continuous chain of irregular clumps (see Figure 4). Most of the cold, high column density clumps in the $\left[l^{-}, b^{-}\right]$and $\left[l^{+}, b^{+}\right]$arms of this feature are seen in silhouette against the $70 \mu \mathrm{m}$ background (Figure 1); the same is not true for clumps along the $\left[l^{+}, b^{-}\right]$and $\left[l^{-}, b^{+}\right]$arms. We conclude that the $\left[l^{-}, b^{-}\right]$and $\left[l^{+}, b^{+}\right]$arms are in front of the bulk of the warmer dust emission (i.e., between us and the Galactic center), while the $\left[l^{-}, b^{-}\right]$and $\left[l^{+}, b^{+}\right]$arms are located in the background.

We use the CS survey by Tsuboi et al. (1999, their Figure 4) to extract the radial velocity of CS condensations positionally coincident with the cold and dense peaks on various positions along the $\infty$-shaped feature in Figures 3 and 4. The position-velocity information is averaged in latitude bins of 1'.5, so the information can be extracted with some degree of approximation but should be adequate to represent the mean velocity of the various clouds and clumps. The full span of velocities found toward each position is reported in Figure 4.

The $\left[l^{-}, b^{+}\right]$arm shows steadily increasing (in absolute values) negative velocities from the center of symmetry toward Sgr C indicating approaching material with increasing relative speed. Following the $\left[l^{-}, b^{-}\right]$arm from $\mathrm{Sgr} \mathrm{C}$ back to the center, we find negative velocities decreasing and changing to positive (receding) values at $l \sim 359^{\circ} .8$. Continuing along the $\left[l^{+}, b^{+}\right]$ arm the radial velocities are still positive and slowly increasing, indicating material receding at higher and higher speeds up to Sgr B2. Finally along the $\left[l^{+}, b^{-}\right]$arm we see velocities decrease again going back toward the center of symmetry of the $\infty$-shaped feature. The distribution of radial velocities along the $\infty$-shaped feature seems to indicate an ordered, rotating pattern around its projected center of symmetry.

\subsection{A Simple Model for the $\infty$-shaped Feature: A 100 pc Elliptical Twisted Ring}

A simplified toy model of the $\infty$-shaped feature is built assuming that the material is distributed along an elliptical orbit (projected onto the Galactic Plane) with semi-axes $[a, b]$ and major-axis position angle $\theta_{p}$. For simplicity, a constant orbital speed $v_{\text {orb }}$ is assumed. An additional sinusoidal vertical oscillation component with a vertical frequency $v_{z}$ and a phase $\theta_{z}$ is added to the equations of the ellipse describing the orbit. Adopting a coordinate system with the $x$-axis oriented toward negative longitudes, the $y$-axis pointing away along the line of sight from the Sun, and the $z$-axes toward positive latitudes (see Figure 5), the position and radial velocity of each point along the orbit are described as a function of the polar angle $\theta_{t}$ (counterclockwise from the $x$-axes) as

$$
\left\{\begin{array}{l}
x=a \cos \theta_{t} \cos \theta_{p}-b \sin \theta_{t} \sin \theta_{p} \\
y=a \cos \theta_{t} \sin \theta_{p}+b \sin \theta_{t} \cos \theta_{p} \\
z=z_{0} \sin v_{z}\left(\theta_{p}-\theta_{z}\right) \\
v_{r}=-v_{\text {orb }} \cos \left(\theta_{p}+\theta_{t}\right) .
\end{array}\right.
$$

We let $a, b, \theta_{p}$, and $v_{\text {orb }}$ vary until they match the observed projected morphology of the ellipse and the distribution of radial velocities at 20 positions along the $\infty$-shaped feature. We keep $z_{0}$ fixed at $15 \mathrm{pc}$, which is half of the measured $b$-extent of the $\infty$-shaped feature; we also fix $v_{z}=2$ in units of the orbital frequency, as indicated by the projected twist shape (see Section 3.3). The fitting procedure minimizes a pseudo- $\chi^{2}$ value $\xi$ :

$$
\xi=\sum_{i=1}^{20} \frac{\left(v_{r}-v_{\mathrm{obs}}\right)^{2}}{\Delta v_{\mathrm{obs}}}
$$




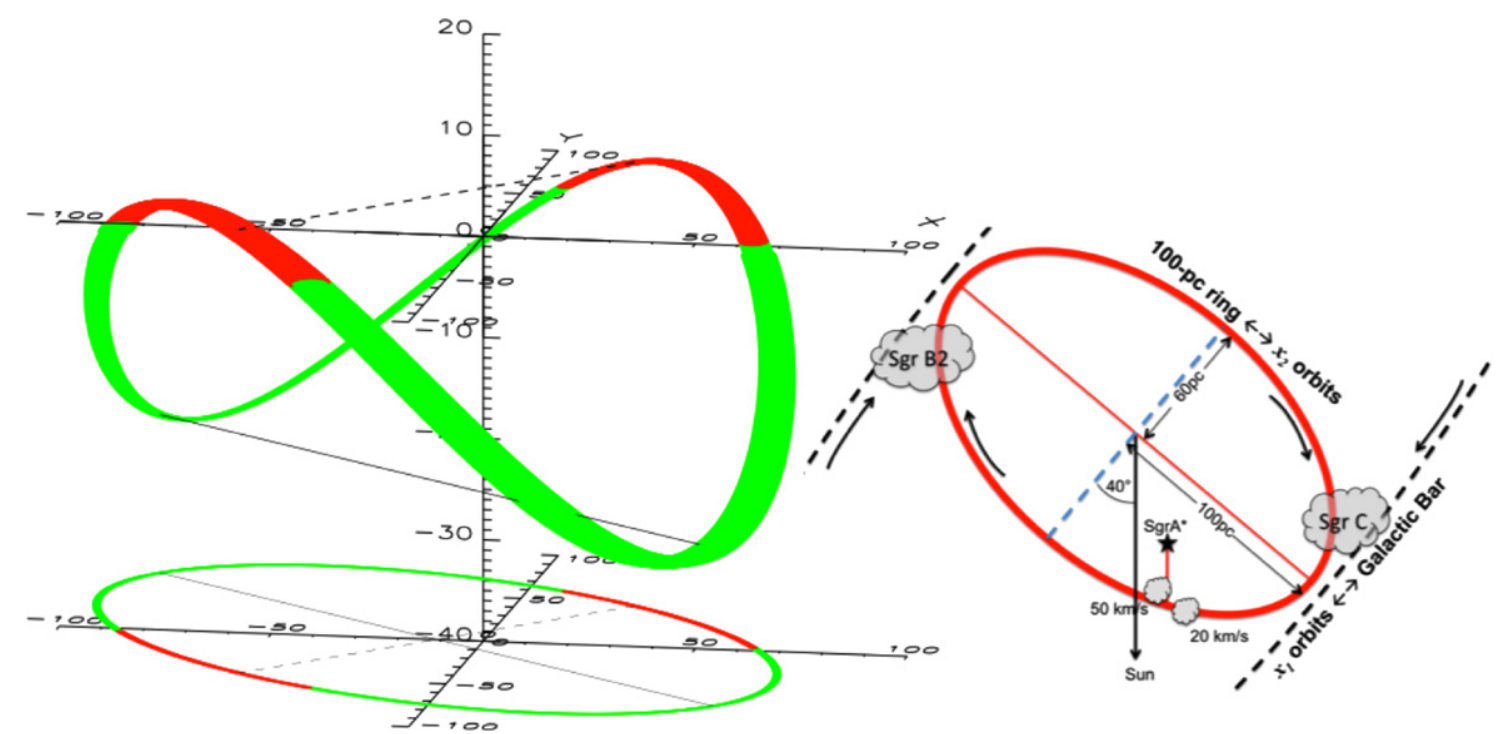

Figure 5. Sketch of the proposed three-dimensional structure and placement of the $100 \mathrm{pc}$ ring. Left panel: the ring is represented by the thick color line (red and green mark positions above and below $b=0^{\circ}$ ). The line of sight to the Sun is along the $Y$-axes. The thin full and dashed lines represent the major and minor axes of the ellipse. Right panel: top view of the $100 \mathrm{pc}$ ring with the proposed location of major clouds. The thick black dashed lines represent the innermost $x_{1}$ orbits. The position of Sgr A* along the line of sight is the one corresponding to the distance it should have from the front portion of the 100 pc ring to justify the velocity difference between the 20 and $50 \mathrm{~km} \mathrm{~s}^{-1}$ clouds if due to the gravitational pull of the mass concentration around Sgr $\mathrm{A}^{\star}$ (see the text).

where $\Delta v_{\text {obs }}$ is half of the velocity range toward each of the 20 test positions (see Figure 4).

The best fit is obtained for an ellipse with $a=100 \mathrm{pc}$, $b=60 \mathrm{pc}, \theta_{p}=-40^{\circ}$, and $v_{\text {orb }}=80 \mathrm{~km} \mathrm{~s}^{-1}$; we obtain $\theta_{z} \sim 0$ implying that the ring crosses the mid-plane along the $X$-axes. These numbers should be taken with caution, as the velocities are derived by eye from a plot. However, the discrepancy between the model radial velocity and the observed ones is within $\pm 25 \mathrm{~km} \mathrm{~s}^{-1}$. The only exception is the $50 \mathrm{~km} \mathrm{~s}^{-1}$ cloud (see Figure 3 ) located close in projection to $\mathrm{Sgr} \mathrm{A}^{\star}$ (see Section 3.2).

The CMZ has been the subject of many studies in millimeter molecular tracers, and although the interpretation is difficult for the extreme confusion along the line of sight, several features were identified. Sofue (1995) analyzed the CO data in Bally et al. (1987) and identified two large-scale structures (the "Arms"). Tsuboi et al. (1999) recognized a large-scale feature in their CS position-velocity diagram that they call "Galactic Center Bow" which matches the $\left[l^{-}, b^{-}\right]-\left[l^{+}, b^{+}\right]$arms of the $\infty$-shaped feature. The cold, high column density dust features identified in the Herschel images, used as a proxy for the densest molecular gas, now indicate that the previously identified "Arms" and the "Galactic Center Bow" are parts of a single, twisted, and elliptical ring rotating around $\mathrm{Sgr}^{\star}$. Figure 5 presents a sketch of the proposed arrangement of what we will call the $100 \mathrm{pc}$ ring.

A rough estimate of the ring mass is obtained by integrating the $N(\mathrm{H})$ map within a contour surrounding the $100 \mathrm{pc}$ ring which encloses material with $N(\mathrm{H}) \gtrsim 2 \times 10^{23} \mathrm{~cm}^{-2}$ (for a corresponding solid angle of $\sim 4 \times 10^{-5} \mathrm{sr}$ ). The contribution of background or foreground gas is removed by subtracting a column density of $N(\mathrm{H})=2 \times 10^{23} \mathrm{~cm}^{-2}$, the mean $N(\mathrm{H})$ value in the interior of $\infty$-shaped feature. The resulting ring mass is $\sim 3 \times 10^{7} M_{\odot}$ (it is $\sim 4 \times 10^{7} M_{\odot}$ without background subtraction). Within the uncertainties, the derived ring mass agrees with $5.3 \times 10^{7} M_{\odot}$ estimated from SCUBA maps (Pierce-Price et al. 2000) and $3 \times 10^{7} M_{\odot}$ from CO data (Dahmen et al. 1998).
The $100 \mathrm{pc}$ ring traces gas and dust moving about the nucleus on an elliptical orbit whose major axis (the straight red line in the right panel of Figure 5) is perpendicular to the major axis of the great Galactic bar, oriented $20^{\circ}-45^{\circ}$ with respect to our line of sight (see Figure 5). The $100 \mathrm{pc}$ ring is likely located on the so-called $x_{2}$ orbit system, which precesses at the same rate as the solid-body angular velocity of the Galactic Bar, resulting in stable, non-intersecting trajectories (Binney et al. 1991). These $x_{2}$ orbits are enclosed within another larger system of stable orbits, called $x_{1}$, elongated along the Galactic Bar. The prominent Sgr B2 and Sgr C star-forming complexes are located at the two projected longitude extrema of the $100 \mathrm{pc}$ ring where gas on $x_{1}$ and $x_{2}$ orbits may collide, producing strong shocks which may trigger the formation of massive molecular cloud formation as well as enhanced star formation rates by the shock focusing mechanism suggested by Kenney \& Lord (1991) for the spiral-bar interface of M83.

It is tempting to speculate that the $100 \mathrm{pc}$ ring constitutes the remnant of a more persistent dusty torus that may have played a role in past active galactic nucleus (AGN) phases of our Galaxy. The recent Fermi-LAT detection of a large gamma-ray bubble emanating from the Galactic center (Su et al. 2010; Crocker 2011; Zubovas et al. 2011) provides possible evidence for such past activity. Mid-IR interferometry of AGNs reveals compact dusty structures with a radius of a few parsecs (e.g., Jaffe et al. 2004; Radomski et al. 2008), but there is additional evidence from Spitzer imaging and spectroscopy suggesting that larger and more persistent structures play a role in shaping observed AGN properties (e.g., Shi et al. 2006; Quillen et al. 2006). Model predictions (e.g., Krolik 2007) for torus column density in excess of $10^{24} \mathrm{~cm}^{-2}$, and orbital speed $\sim 100 \mathrm{~km} \mathrm{~s}^{-1}$, seem to be consistent with the parameters we estimate for the $100 \mathrm{pc}$ ring.

\subsection{The Relation Between the 100 pc Ring and Sgr $\mathrm{A}^{\star}$}

Sgr $\mathrm{A}^{\star}$ is noticeably displaced $\sim 10^{\prime}$ ( $24 \mathrm{pc}$ in projection) toward the negative longitude side of the geometrical center of 
symmetry of the $100 \mathrm{pc}$ ring. The ring displacement with respect to the supposed location of the Galactic center is consistent with the overall displacement of dense gas within the CMZ. Two-thirds to three-quarters of the cold interstellar gas and dust within the inner few hundred parsecs of the nucleus is at positive longitudes with respect to Sgr A* (Bally et al. 1988; Morris \& Serabyn 1996). A similar asymmetry is present in the observed radial velocity on the $100 \mathrm{pc}$ ring close to the location of Sgr B2, as opposed to Sgr C (see Figure 4). This may indicate an additional pattern motion of $\sim 10-20 \mathrm{~km} \mathrm{~s}^{-1}$ of the $100 \mathrm{pc}$ ring as a whole along its major axes and toward us, implying a "sloshing" motion back and forth that could be explained as the response of the gas to an $m=1$ mode in the Galactic bar potential (Morris \& Serabyn 1996).

The projected proximity of the 20 and $50 \mathrm{~km} \mathrm{~s}^{-1}$ clouds to Sgr $A^{\star}$ has led to the suggestion (e.g., Herrnstein \& Ho 2005) that they are physically interacting with $\mathrm{Sgr} \mathrm{A}^{\star}$. If they are part of the $100 \mathrm{pc}$ ring, then Sgr $\mathrm{A}^{\star}$ must be closer to the front of the ring than to its back; Sgr $A^{\star}$ may then not be in the ring center, nor lie along the ellipse axes. Proximity to $\mathrm{Sgr} \mathrm{A}^{\star}$ could be the source of the roughly $40 \mathrm{~km} \mathrm{~s}^{-1}$ deviation of the radial velocity of the $50 \mathrm{~km} \mathrm{~s}^{-1}$ cloud from the value predicted by the model of the rotating elliptical ring.

Given the extent of the 100 pc ring, its orbital motion is driven by the enclosed gas and bulge stars. If, however, ring material passes in proximity of $\operatorname{Sgr} \mathrm{A}^{\star}$, the gravitational pull due to the strong mass concentration can be substantial. Indeed, the $3.6 \times 10^{6} M_{\odot}$ black hole in Sgr $A^{\star}$ dominates the gravitational potential only to a radius of a few parsecs. Beyond that distance the potential is dominated by the stellar mass in the bulge with a radial density profile of the form $\rho(r) \approx \rho_{0}\left(r / r_{b}\right)^{-\alpha}$, where $\alpha \approx 1.9, r_{b} \sim 0.34 \mathrm{pc}$, and $\rho_{0} \sim 2.1 \times 10^{6} M_{\odot} \mathrm{pc}^{-3}$ (Trippe et al. 2008). We then treat the gravitational pull from the Sgr $A^{\star}$ mass concentration as an additional component over the general rotational motion. Using Newton's second law:

$$
\frac{G M_{C}(r)}{r^{2}}=\frac{\Delta v}{t_{0}},
$$

where $r$ is the distance between $\operatorname{Sgr} \mathrm{A}^{\star}$ and the two molecular clouds (that is assumed similar for the two clouds if they belong to the $100 \mathrm{pc}$ ring), $M_{C}(r)$ is the mass enclosed in a sphere of radius $r$ centered on $\mathrm{Sgr} \mathrm{A}^{\star}$, and $t_{0}$ is the timescale for the cloud passing in front of $\operatorname{Sgr} \mathrm{A}^{\star}$. The latter can be written as $t_{0}=\Delta x / v_{\text {orb }}$, where $\Delta x$ is the projected distance between the 50 and $20 \mathrm{~km} \mathrm{~s}^{-1}$ clouds and $v_{\text {orb }}$ is their orbital speed. Equation (2) can then be rewritten as

$$
r=\sqrt{\frac{G M_{C}(r) \Delta x}{\Delta v v_{\mathrm{orb}}}} .
$$

Using this formulation to estimate $M_{C}(r)$, the measured projected distance between the nearest edges of the two clouds of $\Delta x \sim 7 \mathrm{pc}$, and assuming an orbital speed of $80 \mathrm{~km} \mathrm{~s}^{-1}$ from our toy model fit, Equation (3) provides $r \sim 20 \mathrm{pc}$. In other words, to explain the difference in radial velocities between the 20 and $50 \mathrm{~km} \mathrm{~s}^{-1}$ clouds would require that $\mathrm{Sgr} \mathrm{A}^{\star}$ is closer than $20 \mathrm{pc}$ to the foreground arm of the $100 \mathrm{pc}$ ring.

\subsection{The Ring's Twist: Constraints on the Flattening of the Galactic Potential}

Perhaps the most striking aspect of the $100 \mathrm{pc}$ ring is its twist. Apparently, the gas in the ring oscillates twice about the mid-plane each time it orbits the nucleus once. Assuming the elliptical axes and the orbital speed derived from the toy model, the orbital period is $P_{o} \sim 3 \times 10^{6}$ years; the period of the vertical oscillations is therefore $P_{z} \sim 1.5 \times 10^{6}$ years. The coherence of the ring's morphology suggests stability over a few revolutions. Thus, the relationship between $P_{o}$ and $P_{z}$ can be used to infer constraints on the flattening of the gravitational potential in the CMZ. Assuming as a gross approximation that the matter (gas and stars) is distributed in a uniform-density $\rho_{0}$ slab of thickness $h, P_{o}$ at a distance $R$ can then be expressed as

$$
P_{o}=\sqrt{\frac{4 \pi R}{G \rho_{0} h}} .
$$

A perturbation on a particle rotating in the flattened slab potential will result, in a first-order approximation (Binney \& Tremaine 1987), in a harmonic oscillation both in radial and vertical directions. The vertical oscillation frequency can be expressed as

$$
v_{z}^{2}=\left(\frac{\partial^{2} \Phi}{\partial z^{2}}\right)_{R, 0} .
$$

The gravitational field along the $z$-direction of the slab (approximated as infinite) is

$$
F_{z}=2 \pi \rho_{0} G h,
$$

so that the period of the vertical oscillations is

$$
P_{z}=\sqrt{\frac{2 \pi}{\rho_{0} G}} .
$$

As noted above, the $\infty$ shape that the ring displays in projection implies that

$$
P_{o}=2 P_{z}
$$

or, using Equations (4) and (7),

$$
h=\frac{R}{2} \text {. }
$$

The shape of the $100 \mathrm{pc}$ ring would therefore imply a flattened potential in the $\mathrm{CMZ}$ that is consistent with a $x / z=0.5$ axial ratio bulge mass distribution, remarkably similar to the value of $0.5-0.6$ recently derived (Rodriguez-Fernandez \& Combes 2008), fitting the 2MASS data to a potential model that includes the bulge and the nuclear bar.

\section{CONCLUSIONS}

The far-infrared multi-band images provided by the Hi-GAL survey using the Herschel PACS and SPIRE cameras provide evidence for a $100 \times 60$ pc elliptical ring of cold and dense clouds orbiting around the Galactic center on $x_{2}$ orbits elongated along the minor axis of the Galactic center Bar.

The ring is twisted with a vertical frequency twice the orbit frequency, indicating a flattening ratio of 2 for the Galactic center gravitational potential, consistent with the value derived from bulge star 2MASS number counts.

Data processing and map production have been possible thanks to ASI support via contract I/038/080/0. We thank the referee M. Morris for useful comments that led to a more focused paper. We thank L. Spinoglio, N. Sacchi, and A. Marconi for useful discussions on AGNs. We also thank M. Pohlen and the SPIRE ICC for the preliminary analysis on the SPIRE "brightsource mode" calibration. 


\section{REFERENCES}

Bally, J., Stark, A. A., Wilson, R. W., \& Henkel, C. 1987, ApJS, 65, 13

Bally, J., Stark, A. A., Wilson, R. W., \& Henkel, C. 1988, ApJ, 324, 223

Bally, J., et al. 2010, ApJ, 721, 137

Benjamin, R. A., et al. 2003, PASP, 115, 953

Bernard, J. P., et al. 2010, A\&A, 518, L88

Binney, J., Gerhard, O. E., Stark, A. A., Bally, J., \& Uchida, K. I. 1991, MNRAS, 252,210

Binney, J., \& Tremaine, S. 1987, Galactic Dynamics (Princeton Series in Astrophysics; Princeton, NJ: Princeton Univ. Press)

Compiègne, M., et al. 2011, A\&A, 525, 103

Crocker, R. M. 2011, in Proc. 25th Texas Symposium on Relativistic Astrophysics, ed. F. M. Rieger, C. van Eldik, \& W. Hofmann, arXiv:1103.4523

Dahmen, G., Huttemeister, S., Wilson, T. L., \& Mauersberger, R. 1998, A\&A, 331,959

Goto, M., et al. 2008, ApJ, 688, 306

Griffin, M. J., et al. 2010, A\&A, 518, L3

Herrnstein, R. M., \& Ho, P. T. P. 2005, ApJ, 620, 287

Jaffe, W., et al. 2004, Nature, 429, 47

Kenney, D. P., \& Lord, S. D. 1991, ApJ, 381, 118

Kormendy, J., \& Cornell, M. E. 2004, in Penetrating Bars Through Masks of Cosmic Dust: the Hubble Tuning Fork Strikes a New Note, ed. D. L. Block et al. (Astrophysics and Space Science Library, Vol. 319; Dordrecht: Kluwer), 261

Kormendy, J., \& Kennicutt, R. C., Jr. 2004, ARA\&A, 42, 603

Krolik, J. H. 2007, ApJ, 661, 52

Minchev, I., Nordhaus, J., \& Quillen, A. C. 2007, ApJ, 664, L31
Molinari, S., Faustini, F., Schisano, E., Pestalozzi, M., \& Di Giorgio, A. 2010a, A\&A, submitted

Molinari, S., et al. 2010b, PASP, 122, 314

Morris, M., \& Serabyn, E. 1996, ARA\&A, 34, 645

Oka, T., Geballe, T. R., Goto, M., Usuda, T., \& McCall, B. J. 2005, ApJ, 632, 882

Pierce-Price, D., et al. 2000, ApJ, 545, L121

Pilbratt, G., et al. 2010, A\&A, 518, L1

Poglitsch, A., et al. 2010, A\&A, 518, L2

Quillen, A. C., Brookes, M. H., Keene, J., Stern, D., Lawrence, C. R., \& Warner, M. W. 2006, ApJ, 645, 1092

Radomski, J. T., et al. 2008, ApJ, 681, 141

Reid, M. J., et al. 2009, ApJ, 700, 137

Rodriguez-Fernandez, N. J., \& Combes, F. 2008, A\&A, 489, 115

Rodríguez-Fernández, N. J., Martín-Pintado, J., Fuente, A., \& Wilson, T. L. 2004, A\&A, 427, 217

Shi, Y., et al. 2006, ApJ, 653, 127

Sodroski, T. J., et al. 1994, ApJ, 428, 638

Sofue, Y. 1995, PASJ, 47, 527

Su, M., Slayter, T. R., \& Finkbeiner, D. P. 2010, ApJ, 724, 1044

Traficante, A., et al. 2011, MNRAS, in press (arXiv:1106.0698)

Trippe, S., et al. 2008, A\&A, 492, 419

Tsuboi, M., Handa, T., \& Ukita, N. 1999, ApJS, 120, 1

Yusef-Zadeh, F., Braatz, J., Wardle, M., \& Roberts, D. 2008, ApJ, 683, L147

Yusef-Zadeh, F., et al. 2009, ApJ, 702, 178

Zubovas, K., King, A. R., \& Nayakshin, S. 2011, MNRAS, in press (arXiv:1104.5443) 